\title{
Scaling up of Low Resolution Images using Super Resolution Techniques \& Performing Intensity Correction for Medical Imaging
}

\author{
${ }^{1}$ Jithin Saji Isaac and ${ }^{2}$ Ramesh Kulkarni \\ Department of Electronics \& Telecommunication, \\ Vivekanand Education Society's Institute of Technology, Mumbai, India \\ 1jithinsaji@gmail.com, ${ }^{2}$ ramesh.kulkarni@ves.ac.in
}

\begin{abstract}
The diagnosis of illness and certain underlying conditions can be effectively done by the use of Medical imaging. Higher resolution images are used to increase the diagnostic capabilities of the medical practitioner which leads to early and effective diagnosis of an ailment. Although a lot of advanced devices like Computerized Tomography (CT), Magnetic Resonance Imaging (MRI) etc. are currently available, the problem of Noise, Blur limits the overall ability of these devices to produce higher resolution images. A solution which can be proposed is the use of Super Resolution (SR) techniques which can be used for processing of such images. In this paper we make use of intensity correction of input medical images. The super resolution methods are done patch wise. The input images are divided into patches and dictionaries containing high and low resolution patches are obtained. Using the k-SVD algorithm for dictionary learning and OMP method for image super resolution reconstruction, the final high resolution image is obtained. The combination of intensity correction and super resolution leads to computationally and visually better results.
\end{abstract}

Keywords: Intensity inhomogeneity, Super Resolution, Dictionary learning, Sparse Representation, MRI

\section{Introduction}

It is desirable to have high resolution images for all our daily applications involving Image processing. With the help of a high resolution image, one can easily increase the accuracy with respect to finding a localized tumor or increase the visual excellence of watching a high definition video. The quality of the sensor generally decides the resolution of the image. But as the quality of the sensor increases, so does the cost of the acquisition device. Therefore a solution needs to be reached to overcome this hardware problem and replace it with a software system which will help to increase the resolution by keeping the hardware same. Such a software system is discussed in this paper and the name of the image processing technique used is called as Super Resolution Reconstruction.

In Image processing, the manipulation of digital images are undertaken. Image enhancing algorithms and software are incorporated to achieve this image enhancement. This high resolution image is used in various different engineering fields to zoom in on a particular region of interest. The raw data from the 
image acquisition sensors are processed further and are given to different image processing and enhancement algorithms to help remove the noise element blur components and increase the resolution of the image.

\section{The Concept of Super Resolution}

Super-resolution basically points out to the concept of combining low resolution and noisy images of a particular region and using these to obtain the higher resolution image.

The basic final aim of Super Resolution (SR) or Super Resolution Reconstruction (SRR) methods is to get the High Resolution (HR) image from one or more Low Resolution (LR) input images. Super Resolution $(\mathrm{SR})$ is done either using the single image method wherein one low resolution image is used to obtain the higher resolution image using a dictionary method or is done using the multiple image method wherein multiple low resolution images are used to obtain the final high resolution image. [12]

The advantage of the single image based super resolution method is that we do not require many LR images. We use only one LR image and divide these into patches and work on one specific patch to obtain the higher resolution patch. The higher resolution patches are then used to find out the final high resolution images by combining all the higher resolution patches together.

We resample the high resolution image and produce the low resolution image. To this low resolution image we apply the Super Resolution algorithms which include the steps of Interpolation, noise and blur removal. When these steps are applied to the patch of the sampled LR image, we receive the HR patch. These HR patches later on form the HR image.

\section{Super Resolution in Medical Images}

Normal interpolation techniques are found for medical images consisting of intensity inhomogenity, blur and additive noise. Simple techniques like interpolation can only add extra pixels and improve the resolution but not do any task of removal of noise and intensity corrections. Such problems give rise to the new methods of Super Resolution wherein along with image resolution, the quality of the image with respect to noise and blur removal also needs to be considered.

Medical images are unique for the particular reason that they are taken in an environment which is much more challenging than the normal acquisition atmosphere. Therefore, the problems that accompany the medical images are very much of a higher magnitude when compared to normal images. General problems plaguing the medical images are given as below: [12]

- Low resolution

- Higher magnitude of Noise component

- Images with low contrast

- Imaging artefacts present in the image

\section{Intensity Correction of Medical Images}

Variations of illumination in the spatial domain and the imperfections of devices that capture the images can lead to many problems in Computer vision and Image processing techniques. The particular cases of Image segmentation may be difficult for images that have intensity inhomogenity due to different ranges of intensity that exist in an inhomogeneous image, thus making it difficult to identify images 
Jithin Saji Isaac and Ramesh Kulkarni; Scaling up of Low Resolution Images using Super Resolution Techniques \& Performing Intensity Correction for Medical Imaging. Journal of Biomedical Engineering and Medical Imaging, Volume 2, No 6, December (2015) , pp 99-108

based on image intensity. Present day algorithms rely on the homogeneity of intensity and therefore images that are inhomogeneous are not applicable for practical purposes. Therefore, the idea of having level intensity homogeneity is very important. [10]

In this paper, a novel region-based method for image segmentation is used. This paper makes use of a local intensity clustering property and defines a local area clustering benchmark function for the intensities in a neighbourhood of each point. An accepted model of images with intensity inhomogeneities is used. This local area clustering benchmark is integrated over the intensity centre to define an energy functional. This energy function is then converted into a level set formulation. By means of bias field estimation and energy minimization, a level set evolution of intensity is achieved.

Bias correction and segmentation of MRI images can be extensively done with this method. This method is based on a model of an image which describes a combination of real world images which defines intensity inhomogenity to be a part of the image. By using the multiplicative model of intensity in homogeneity, we can observe a given as a combination of the original image as the first part, the second part that consists of intensity in homogeneity and the third part that consists of additive noise. The component that gives an observation of the pixels that have high intensity is referred to as a bias field (or shading image). This field we assume to be slowing varying. The additive noise is assumed to be zero-mean Gaussian noise. The image segmentation methods based on region selection typically relies on a specific region description of the intensities in each region to be segmented. But it becomes very difficult to give a descriptor for images which have intensity in homogeneities. As a result it gets very difficult to segment the inhomogeneous regions directly based on the different intensities of pixels involved in the image.

Here a variation level set criterion for image segmentation and image bias correction with intensity in homogeneity is used. [10] The twin tasks of Segmentation and bias field exploration is jointly done by reducing the proposed energy functional. This method is much better than piecewise smooth model used earlier. An experimental result on the MRI of a shoulder and MRI image of a brain gives superior results which can be seen in the below figure. Effective Image segmentation and bias estimation of the MRI image occurs and we observe an intensity homogenous image as under.

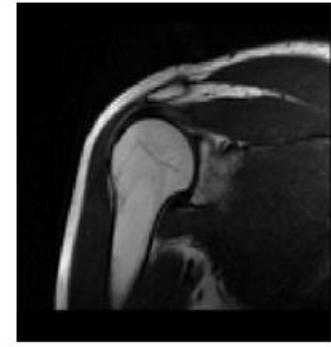

(a)

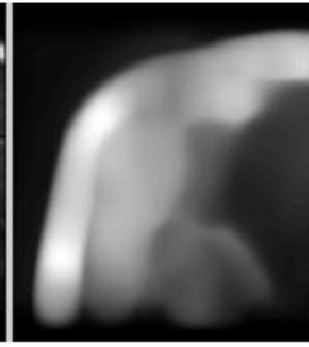

(b)

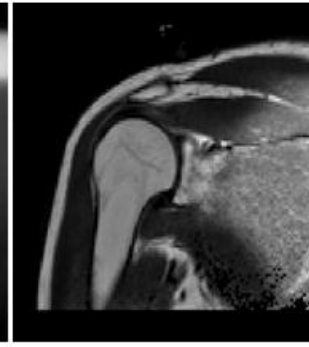

(c)

Figure. 1 Intensity Correction MRI image of shoulder: Original image of MRI of shoulder (a), bias field of the image (b) and Intensity corrected image (c) 


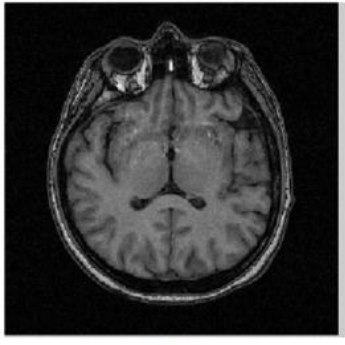

(a)

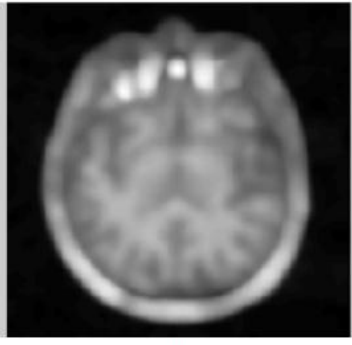

(b)

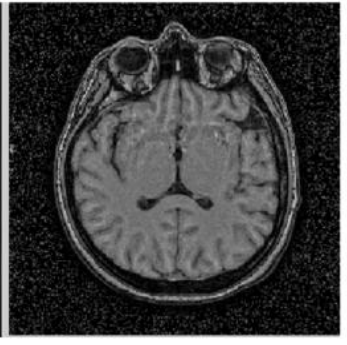

(c)

Figure. 2 Intensity Correction MRI image of shoulder: Original image of MRI of shoulder (a), bias field of the image (b) and Intensity corrected image (c)

\section{Existing Technologies \& Proposed Algorithm for Super Resolution of Medical Images}

We can write the single image scale of problem as follows:

- Denote the original high-resolution image as $\mathrm{yh}_{\mathrm{h}}$.

- Denote the blur and decimation operators as $\mathrm{H}$ and S.

- $\mathrm{H}$ is a low-pass filtering which we perform on the image and $\mathrm{S}$ is the decimation of the image which we perform by an integer factor s.

- $\quad z_{l}$ is the Noisy and low-resolution image obtained from the version of the original image $y_{\mathrm{h}}$

Therefore, we denote the low resolution image obtained as $\mathrm{z}_{\mathrm{l}}$.

$$
\mathrm{z}_{\mathrm{l}}=\mathrm{SH} \mathrm{y}_{\mathrm{h}}+\mathrm{v},
$$

Where $v$ is an Additive i.i.d. White Gaussian noise.

Given $\mathbf{z}_{\mathrm{l}}$, the problem is to find a new image $y$ such that $\mathrm{y} \approx \mathrm{y}_{\mathrm{h}}$. The maximum-likelihood estimation is obtained by the minimization of || $\mathbf{S H y}-\mathbf{z}_{1}||_{2}$ due to the inherent Gaussian nature of the Gaussian noise component $\mathrm{v}$.

The Sparse-Land model is used for the scale-up problem in this paper as was introduced in [7], [8] \& [9]. The basic assumption is that each of the patches from the images selected can be represented as a linear combination of patches from the dictionary i.e. each patch is obtained by multiplying the dictionary by a sparse vector of coefficients.

Often there is a problem in the resolutions of $z_{1}$ and $y_{h}$. To avoid this issue, $z_{1}$ is scaled up by Bicubic interpolation to realize into the same size as $y_{h}$. Let us now call this scaled up image as $y_{1}$ instead of $z_{1}$. Let $Q$ be the operator by which we work on $\mathrm{z}_{1}$. Therefore,

$$
y_{l}=Q \cdot z_{1 .}=Q\left(S H y_{h}+v\right)=\left(Q S H y_{h}+Q v\right)=X \cdot y_{h}+v
$$

By using this algorithm, we can operate on patches extracted from y the corresponding patch from $\mathbf{y}_{\mathrm{h}}$.

The algorithm of the proposed method can be segregated into two main sub phases given below: 
Jithin Saji Isaac and Ramesh Kulkarni; Scaling up of Low Resolution Images using Super Resolution Techniques \& Performing Intensity Correction for Medical Imaging. Journal of Biomedical Engineering and Medical Imaging, Volume 2, No 6, December (2015) , pp 99-108

\subsection{Construction of the Training set and Dictionary Training}

The first task to construct the training set is to collect the High resolution images $\mathrm{y}_{\mathrm{h}}$. We then blur and down scale these images by a factor of $s$. Thus the low resolution images $z_{l}$ are formed. These $z_{1}$ are then scaled up using the $Q$ interpolation factor to $\mathrm{y}_{\mathrm{I}}$. Thus, $\mathrm{y}_{\mathrm{l}}=\mathrm{X} . \mathrm{y}_{\mathrm{h}}+\mathrm{v}$

Next, pairs of matching patches are extracted which will eventually form the training database $P=\left\{p_{h}\right.$, $\mathrm{pl}$. All these patch-pairs individually undergo a pre-processing stage that removes the low frequencies from $p_{h}$ and extracts features from $p_{\text {.. }}$ [2][3]

The very important step of the dictionary learning stage is done using the K-SVD dictionary training proposed in [4]. This algorithm is applied to the patches and we get the LR dictionary $D_{1}$. The residual component of this training procedure is the sparse representation coefficients vectors $\left\{q^{k}\right\}$ that correspond to the training patches $\left\{p_{\mid}\right\}$. To represent the low resolution patches sparsely, the dictionary $D_{1}$ is trained.

Now that the low resolution dictionary is constructed, we need to construct the high resolution dictionary $D_{h}$. Once we get $D_{h}$, then we can get the HR patch $p_{h}$ by approximating it as $p_{h} \approx D_{h} q^{k}$.

\subsection{Super Resolution Reconstruction of Low resolution image into High Resolution Image}

- First the low resolution image $z_{1}$ is scaled to $y_{1}$ using Bicubic interpolation and bought to same size as Yh.

- The image $y_{1}$ is then pre-processed using High pass filters and then patches are extracted from the result of this filtering giving us $\mathrm{p}_{\mathrm{I}}$.

- The OMP algorithm [11] is applied to $p_{1}$ by allocating $L$ atoms to the representation and the sparse representation vector $\mathrm{q}^{\mathrm{k}}$ is obtained.

- $\mathrm{q}^{\mathrm{k}}$ is then multiplied with the high resolution dictionary $D_{h}$ and the approximated HR patch $\mathrm{p}_{h}$ is obtained.

- The final high resolved output $y_{h}$ is constructed from $p_{h}$ by combining all these high resolution patched together.

\subsection{Using Single Image as Dictionary set}

If during the training process of the dictionary, there are no external set of images, then the algorithm is trained to use a single image. This single image will be the same Low resolution image that we input to the system i.e. image $z_{1}$. This image $z_{1}$ is then used as the supposed high resolution image and the scaled down version of this image is called as $z_{l s}$. The images $z_{l}$ and $z_{1 s}$ are then subjected to the dictionary training phase. These trained dictionaries are then used to reconstruct the high resolution image back i.e. to get back $z_{1}$ and ultimately we can scale that up and get $\mathrm{y}_{\mathrm{h}}$.

The following block diagram in figure no. 3 gives a basic pictorial idea of the entire flow of the proposed algorithm of the project. 


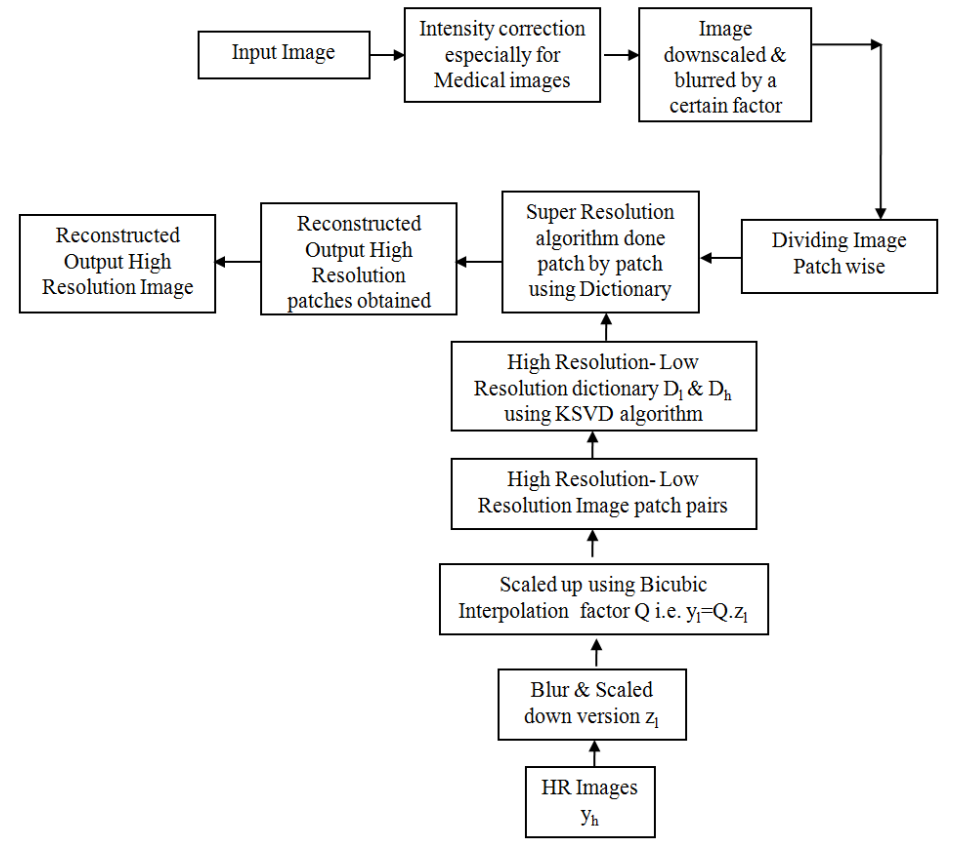

Figure. 3 Basic Block Diagram of the Proposed Method.

\section{Outputs}

The following outputs were achieved for the flow chart of the proposed algorithm shown above. The input to the sparse representation method and proposed method is the resized down scaled input image, in this case, it is the MRI image of the shoulder.

The fig. 4 shows the resized image of the original image. This image is given to the Bicubic, Sparse and the proposed Image upscale algorithm. The outputs obtained and the corresponding PSNR and SSIM are mentioned below in Table No I.

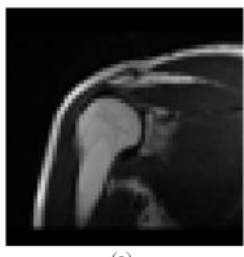

(a)

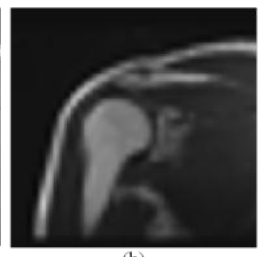

(b)

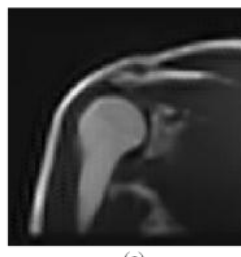

(c)

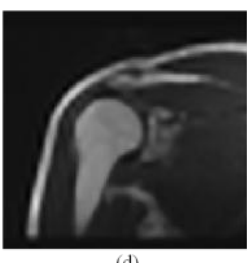

(d)

Figure. 4 Super Resolution of MRI image of shoulder: Input Resized image (a), Output of Bicubic Interpolation

(b), Output of Sparse Representation (c) and Output of Proposed Image scale up algorithm (d)

Table 1: comparing the PSNR \& SSIM values for Bicubic interpolation, sparse representation and proposed image scale up algorithm

\begin{tabular}{|c|c|}
\hline TECHNIQUE & PSNR (dB) \\
\hline Bicubic Interpolation & 27.9 \\
\hline Sparse representation (ScSR) & 29.7 \\
\hline Proposed Image scale up algorithm & 30.5 \\
\hline
\end{tabular}


Jithin Saji Isaac and Ramesh Kulkarni; Scaling up of Low Resolution Images using Super Resolution Techniques \& Performing Intensity Correction for Medical Imaging. Journal of Biomedical Engineering and Medical Imaging, Volume 2, No 6, December (2015) , pp 99-108

Figure No. 5 shows the output of the three methods when Intensity correction is performed on the input image prior to it being downscaled and given to the super resolution stages.

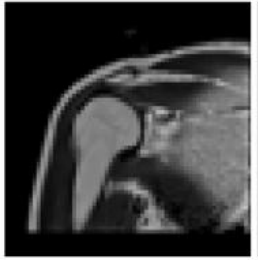

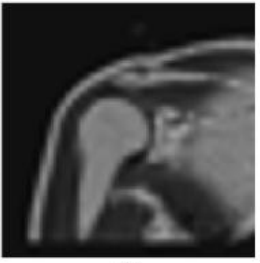

(b)

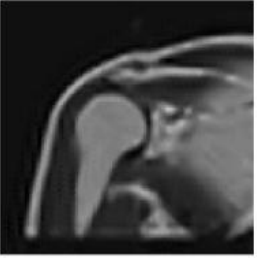

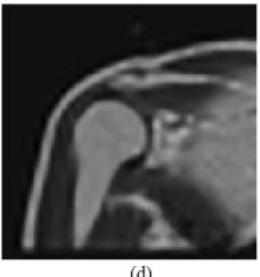

Figure. 5 Super Resolution of MRI image of shoulder: Input resized scaled down image with Intensity correction performed (a), Output of Bicubic Interpolation (b), Output of Sparse Representation (c) and Output of Proposed Image scale up algorithm (d)

Now, before the intensity correction is done, the image is subjected with Gaussian Noise to replicate the real world effect which is bound to occur while capturing medical images. The PSNR \& SSIM of the images subjected to Gaussian Noise \& Intensity Correction are shown Table II and the corresponding images capture in Figure No. 6.

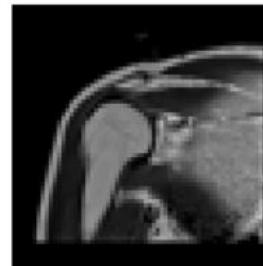

(a)

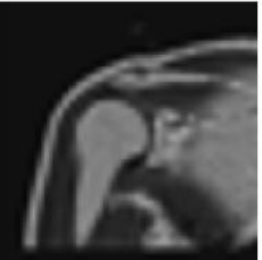

(b)

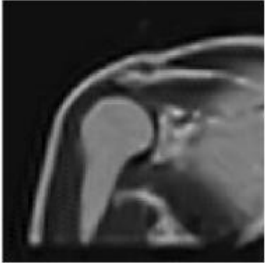

(c)

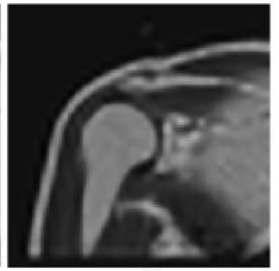

(d)

Figure 6 Effect of adding Gaussian Noise to an Image and then image correcting and super resolving it

Table2: comparing the PSNR \& SSIM values for the different methods when the image is subjected to Gaussian noise

\begin{tabular}{|c|c|}
\hline TECHNIQUE & PSNR (dB) \\
\hline Bicubic Interpolation & 19.9 \\
\hline Sparse representation (ScSR) & 20.2 \\
\hline Image Scale up algorithm & 20.3 \\
\hline
\end{tabular}

The intensity corrected \& Gaussian noise added image in now given to an averaging filter to check for certain changes in the output. Therefore, before the super resolution stage, the resized and intensity corrected image is given to an averaging filter. The basic functional block diagram of the proposed system is shown in Figure No. 7. The corresponding outputs are shown in Figure no. 8 and PSNR and SSIM values are mentioned in Table No. III. 


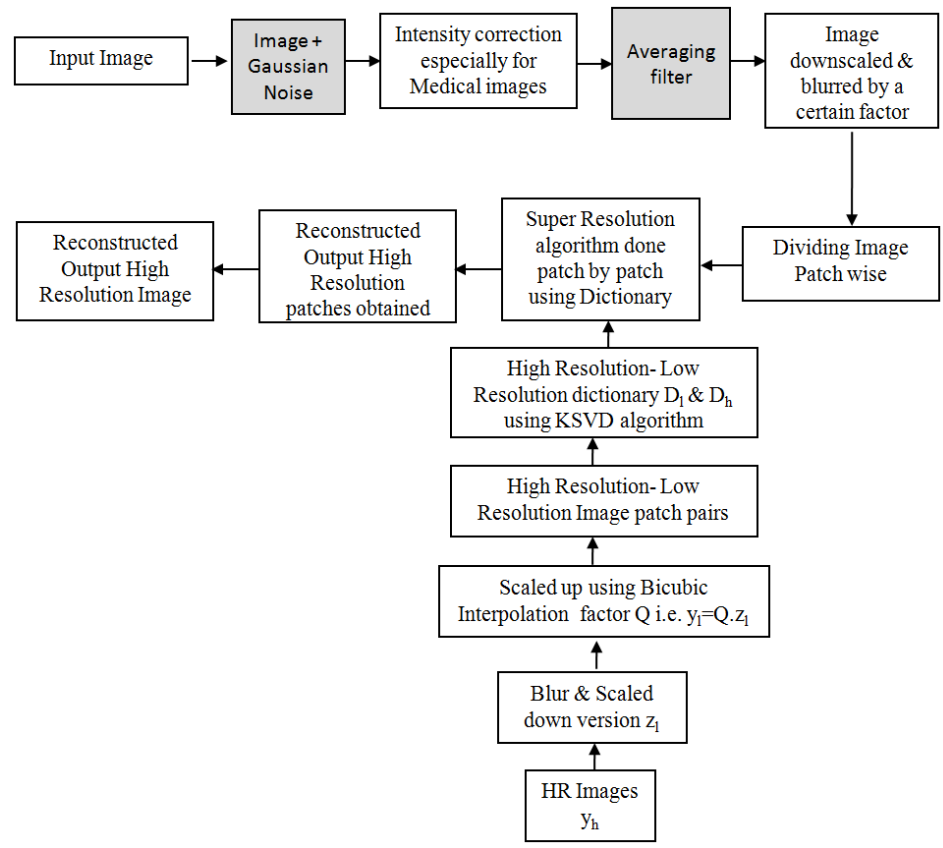

Figure. 7 Basic Block Diagram of the Proposed Method for an image added with Gaussian noise and then passed through an Averaging filter just before the super resolution stage

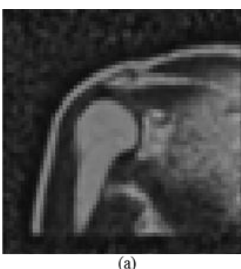

(a)

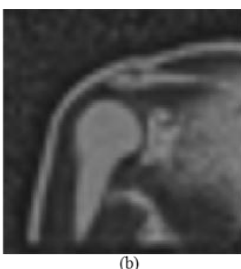

(b)

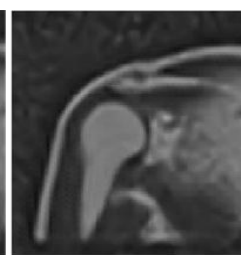

(c)

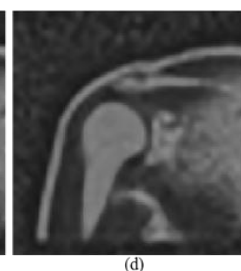

(d)

Figure. 8 Effect of using Averaging filter just before the super resolution stage for partially removing the effects of the Gaussian Noise Added to the image

Table 3: Comparing the PSNR \& SSIM values when the image subjected to Gaussian Noise is passed through an averaging filter before giving it to the Super Resolution Methods

\begin{tabular}{|c|c|}
\hline TECHNIQUE & PSNR (dB) \\
\hline Bicubic Interpolation & 22.9 \\
\hline Sparse representation (ScSR) & 24.4 \\
\hline Image Scale up algorithm & 26.2 \\
\hline
\end{tabular}

\section{Conclusion}

In this paper, the basic work of super resolution is carried out with the help of image patches. Low resolution image patches are converted into high resolution image patches. The detailed work of Yang et. al. in [2] \& [3] forms the cornerstone of all research into the subject of sparse representation. The input image is first intensity corrected before applying to the super resolution phase. This intensity correction forms the special part of this paper. Intensity correction leads to better medical images which are visually and mathematically far better than other images which have intensity inhomogenity. Once the image intensity is performed, the image is given to the super resolution process wherein the image is divided into number of patches. Prior to this, a dictionary consisting of low resolution and high 
Jithin Saji Isaac and Ramesh Kulkarni; Scaling up of Low Resolution Images using Super Resolution Techniques \& Performing Intensity Correction for Medical Imaging. Journal of Biomedical Engineering and Medical Imaging, Volume 2, No 6, December (2015) , pp 99-108

resolution patches is separately trained. Based on this dictionary and the k-SVD algorithm, the dictionary is properly trained. By using the OMP algorithm, the super resolution reconstruction occurs. The high resolution patches are formed which then combine to give us the final high resolution image.

Obtaining a High resolution image is of paramount importance for medical images. A high resolution medical image is necessary for better diagnosis of the ailment. Therefore the concept of Super Resolution is of great value for overcoming the problem of low resolution.

The future scope in the development of SR techniques is tremendous. One can develop newer and better algorithms for the continuous enhancement of the image parameters like PSNR and SSIM, thereby making this field challenging and evolving.

\section{REFERENCES}

[1] S. Chaudhari, Department of Electrical Engineering, Indian Institute of Technology - Bombay, Mumbai, India 400 076, "Super Resolution Imaging". New York, Boston, Dordrecht, London, Moscow, Kluwer Academic Publishers.

[2] J. Yang, J. Wright, T. Huang, Y Ma, "Image super-resolution as sparse representation of raw image patches", IEEE Computer Vision and Pattern Recognition (CVPR) (June 2008)

[3] J. Yang, J. Wright, T. Huang, Y Ma, "Image Super-Resolution via Sparse Representation IEEE Transactions on Image Processing", IEEE Transactions On Image Processing, Vol. 19, No. 11, November 2010

[4] T. Peleg, M. Elad, "A Statistical Prediction Model Based on Sparse Representations for Single Image Super-Resolution", IEEE Transactions on Image Processing, Vol. 23, No. 6, June 2014

[5] M. Aharon, M. Elad, A.M. Bruckstein, "The K-SVD: An algorithm for designing of over complete dictionaries for sparse representation", IEEE Transactions on Signal Processing 54(11), 4311-4322 (2006)

[6] Li, R. Huang, Z. Ding, J. C. Gatenby, D. N. Metaxas, J. C. Gore, "A Level Set Method for Image Segmentation in the Presence of Intensity Inhomogeneities With Application to MRI", IEEE Transactions On Image Processing, Vol. 20, No. 7, July 2011

[7] Trinh, M. Luong, F. Dibos, J.M. Rocchisani, C.D. Pham and T.Q. Nguyen, "Novel Example-Based Method for Super-Resolution and Denoising of Medical Images", IEEE Transactions on Image processing, Vol. 23, No. 4, April 2014

[8] M. Elad, M. Aharon, "Image denoising via learned dictionaries and sparse representation", International Conference on Computer Vision and Pattern Recognition, New York, June 17-22 (2006)

[9] M. Elad, M. Aharon, "Image denoising via sparse and redundant representations over learned dictionaries", IEEE Transactions on Image Processing 15(12), 3736-3745(2006). 
Journal of Biomedical Engineming and Medical Imaging, Volume 2, Issue 6, Dec, 2015

[10] M. Bruckstein, D.L. Donoho, M. Elad, "From Sparse Solutions of Systems of Equations to Sparse Modelling of Signals and Images", SIAM Review 51(1), 34-81(2009)

[11] H. Okuhata, R. Imai, M. Ise, R. Y. Omaki1, H. Nakamura, S. Hara and I. Shirakawa, "Implementation of Dynamic-Range Enhancement and Super-Resolution Algorithms for Medical Image Processing" in 2014 IEEE International Conference on Consumer Electronics (ICCE)

[12] J. S. Isaac, R. Kulkarni, "Super Resolution Techniques for Medical Image Processing", International Conference on Technologies for Sustainable Development (ICTSD), 4-6 February, 2015 